\title{
Erratum
}

\section{GOING, GOING, GONE! A SWIFT TOUR OF AUCTION THEORY AND ITS APPLICATIONS}

\author{
EMIEL MAASLAND* AND SANDER ONDERSTAL**
}

De Economist (2006) 154:197-249

DOI $10.1007 / \mathrm{s} 10645-006-9002-5$

In the original publication of this article, the second formula in the Proof of Proposition 6 in the Appendix was incorrect. Formulae (A.13), (A.15), (A.16) and (A.18) were incorrect as well. The correct formulae are given below.

$$
\begin{aligned}
& \frac{\partial U}{\partial b_{1}}=\left(v_{1}-b_{1}\right) f\left(b^{-1}\left(b_{1}\right)\right)\left(b^{-1}\left(b_{1}\right)\right)^{\prime}-\left(1-F\left(b^{-1}\left(b_{1}\right)\right)\right) \\
& +b_{1} f\left(b^{-1}\left(b_{1}\right)\right)\left(b^{-1}\left(b_{1}\right)\right)^{\prime}=0 . \\
& U_{0}(p, x)=\sum_{i=1}^{n} \int_{\mathrm{V}} v_{i} p_{i}(\mathbf{v}) g(\mathbf{v}) d \mathbf{v}-\sum_{i=1}^{n} \int_{\underline{v}_{i}}^{\bar{v}} U_{i}\left(p, x, v_{i}\right) f_{i}\left(v_{i}\right) d v_{i} . \\
& U_{i}\left(\alpha_{i}\right)=E_{\alpha_{-i}}\left[t_{i}(\boldsymbol{\alpha})-x_{i}(\boldsymbol{\alpha})\left\{\varphi\left(e_{i}(\boldsymbol{\alpha})\right)-\alpha_{i} e_{i}(\boldsymbol{\alpha})\right\}\right],
\end{aligned}
$$

where

$$
\begin{aligned}
& \varphi(e)=\frac{1}{2} e^{2}+e . \\
& \begin{aligned}
U_{i}\left(\alpha_{i}, \tilde{\alpha}_{i}\right)= & E_{\alpha_{-i}}\left[t_{i}\left(\alpha_{-i}, \tilde{\alpha}_{i}\right)-x_{i}\left(\alpha_{-i}, \tilde{\alpha}_{i}\right) \varphi\left(e_{i}\left(\alpha_{-i}, \tilde{\alpha}_{i}\right)\right)\right] \\
& +\alpha_{i} E_{\alpha_{-i}}\left[e_{i}\left(\alpha_{-i}, \tilde{\alpha}_{i}\right) x_{i}\left(\alpha_{-i}, \tilde{\alpha}_{i}\right)\right] .
\end{aligned} \\
& \begin{aligned}
\frac{d U_{i}\left(\alpha_{i}\right)}{d \alpha_{i}}= & \left.\frac{\partial U_{i}\left(\alpha_{i}, \tilde{\alpha}_{i}\right)}{\partial \tilde{\alpha}_{i}}\right|_{\tilde{\alpha}_{i}=\alpha_{i}}+E_{\alpha_{-i}}\left[e_{i}(\boldsymbol{\alpha}) x_{i}(\boldsymbol{\alpha})\right] \\
= & E_{\alpha_{-i}}\left[e_{i}(\boldsymbol{\alpha}) x_{i}(\boldsymbol{\alpha})\right] .
\end{aligned}
\end{aligned}
$$

* Erasmus University Rotterdam, P.O. Box 1738, 3000 DR Rotterdam, The Netherlands.

** University of Amsterdam, Roetersstraat 11, 1018 WB Amsterdam, The Netherlands. 